\title{
International multicenter expert survey on endoscopic treatment of upper gastrointestinal anastomotic leaks
}

\section{(๑) $\odot$}

\section{Authors}

Eduardo Rodrigues-Pinto ${ }^{1}$, Alessandro Repici ${ }^{2}$, Gianfranco Donatelli ${ }^{3}$, Guilherme Macedo ${ }^{1}$, Jacques Devière ${ }^{4}$, Jeanin E. van Hooft ${ }^{5}$, Josemberg M. Campos ${ }^{6}$, Manoel Galvao Neto ${ }^{7}$, Marco Silva ${ }^{1}$, Pierre Eisendrath ${ }^{8}$, Vivek Kumbhari ${ }^{9}$, Mouen A. Khashab ${ }^{9, *}$

Institutions

1 Gastroenterology Department, Centro Hospitalar São João, Porto, Portugal

2 Digestive Endoscopy Unit, Humanitas Research Hospital, Rozzano, Milan; Humanitas University, Rozzano, Milan, Italy

3 Department of Gastroenterology and Hepatology, Hôpital Privé des Peupliers Paris, France

4 Department of Gastroenterology Hepatopancreatology, and Digestive Oncology, Erasme University Hospital, Université Libre de Bruxelles, Brussels, Belgium

5 Department of Gastroenterology and Hepatology, Amsterdam University Medical Center, University of Amsterdam, Amsterdam, The Netherlands

6 Department of Surgery, University of Pernambuco, Recife, Pernambuco, Brazil

7 Herbert Wertheim College of Medicine at Florida International University, Department of Surgery, Miami, Florida, United States

8 Department of Gastroenterology \& Hepatology, CHU Saint Pierre, Université Libre de Bruxelles, Brussels, Belgium

9 Department of Medicine and Division of Gastroenterology and Hepatology, The Johns Hopkins Medical Institutions, Baltimore, Maryland, United States

submitted 29.5.2019

accepted after revision 5.8.2019

Bibliography

DOI https://doi.org/10.1055/a-1005-6632

Endoscopy International Open 2019; 07: E1671-E1682

(c) Georg Thieme Verlag KG Stuttgart · New York elSSN 2196-9736

Supplementary material

Online content viewable at:

https://doi.org/10.1055/a-1005-6632

${ }^{*}$ Full list of collaborators ordered alphabetically at the end of the article.
Corresponding author

Eduardo Rodrigues-Pinto, MD, Gastroenterology

Department, Centro Hospitalar São João, Porto. Al. Prof. Hernâni Monteiro 4200 - 319, Porto, Portugal

Fax: +351225513601

edu.gil.pinto@gmail.com

\section{ABSTRACT}

Background and study aims A variety of endoscopic techniques are currently available for treatment of upper gastrointestinal (UGI) anastomotic leaks; however, no definite consensus exists on the most appropriate therapeutic approach. Our aim was to explore current management of UGI anastomotic leaks.

Methods A survey questionnaire was distributed among international expert therapeutic endoscopists regarding management of UGI anastomotic leaks.

Results A total of $44 \%$ of 163 surveys were returned; $69 \%$ were from gastroenterologists and $56 \%$ had $>10$ years of experience. A third of respondents treat between 10 and 19 patients annually. Fifty-six percent use fully-covered self-expandable metal stents as their usual first option; $80 \%$ use techniques to minimize migration; 4 weeks was the most common reported stent dwell time. Sixty percent perform epithelial ablation prior to over-the-scope-clip placement or suturing. Regarding endoscopic vacuum therapy (EVT), $56 \%$ perform balloon dilation and intracavitary EVT in patients with large cavities but small leak defects. Regarding endoscopic septotomy, $56 \%$ consider a minimal interval of 4 weeks from surgery and $90 \%$ consider the need to perform further sessions. Regarding endoscopic internal drainage (EID), placement of two stents and shorter stents is preferred. Persistent inflammation with clinical sepsis was the definition most commonly reported for endoscopic failure. EVT/stent placement and EVT/EID were the therapeutic options most often chosen in patients with previous oncologic surgery and previous bariatric surgery, respectively.

Conclusions There is a wide variation in the management of patients with UGI anastomotic leaks. Future prospective studies are needed to move from an expert- to evidenceand personalization-based care. 


\section{Introduction}

Gastrointestinal leaks are abnormal communications between the intraluminal and extraluminal compartments as a result of a defect in the integrity of the gastrointestinal wall [1]. They usually occur due to defects at surgical suture sites, being associated with a high risk of mortality and morbidity. Leaks are responsible for the majority of surgical mortality [2-4]. In addition, delayed closure of leaks may result in chronic fistulae formation, which are difficult to manage endoscopically.

Prevalence of upper gastrointestinal (UGI) anastomotic leaks has increased in recent years. Leaks related to oncologic surgery leaks have been reported in $8 \%$ to $26 \%$ after distal esophagectomy and in $3 \%$ to $12 \%$ after total gastrectomy [3, 5]; bariatric surgery leaks have been reported in $2 \%$ to $5 \%$ of patients after Roux-en-Y gastric bypass (RYGB) and in $1 \%$ to $2 \%$ after sleeve gastrectomy [6,7].

Treatment of UGI anastomotic leaks remains controversial, as indications for surgical, conservative and endoscopic therapy remain non-standardized. Traditionally, surgical therapy has been the mainstay of treatment for anastomotic leaks; however, it tends to be complex and is plagued by high rates of morbidity [8]. Over the last decade, interventional endoscopy has evolved as an effective and less invasive alternative to primary surgery, changing the management paradigm for UGI leaks. A variety of techniques are currently available to reestablish the continuity of the digestive tract, prevent or treat infection related to the leak, prevent further contamination, drain potential collections, and provide nutritional support [9]. Endoscopic options include stent placement (metallic, plastic and biodegradable), endoscopic vacuum therapy (EVT), endoscopic internal drainage (EID), through-the-scope [TTS] and over-thescope clips [OTSC], endoscopic suturing, endoscopic septotomy plus balloon dilation and tissue sealants [9]. Theoretically, all of these can be used alone or with a multimodality approach, with the approach chosen being tailored to the clinical and morphologic presentation but also largely institutional dependent and based upon availability of devices and accessories.

Even though endoscopic therapy may be associated with an improved outcome and better quality of life, there is no definite
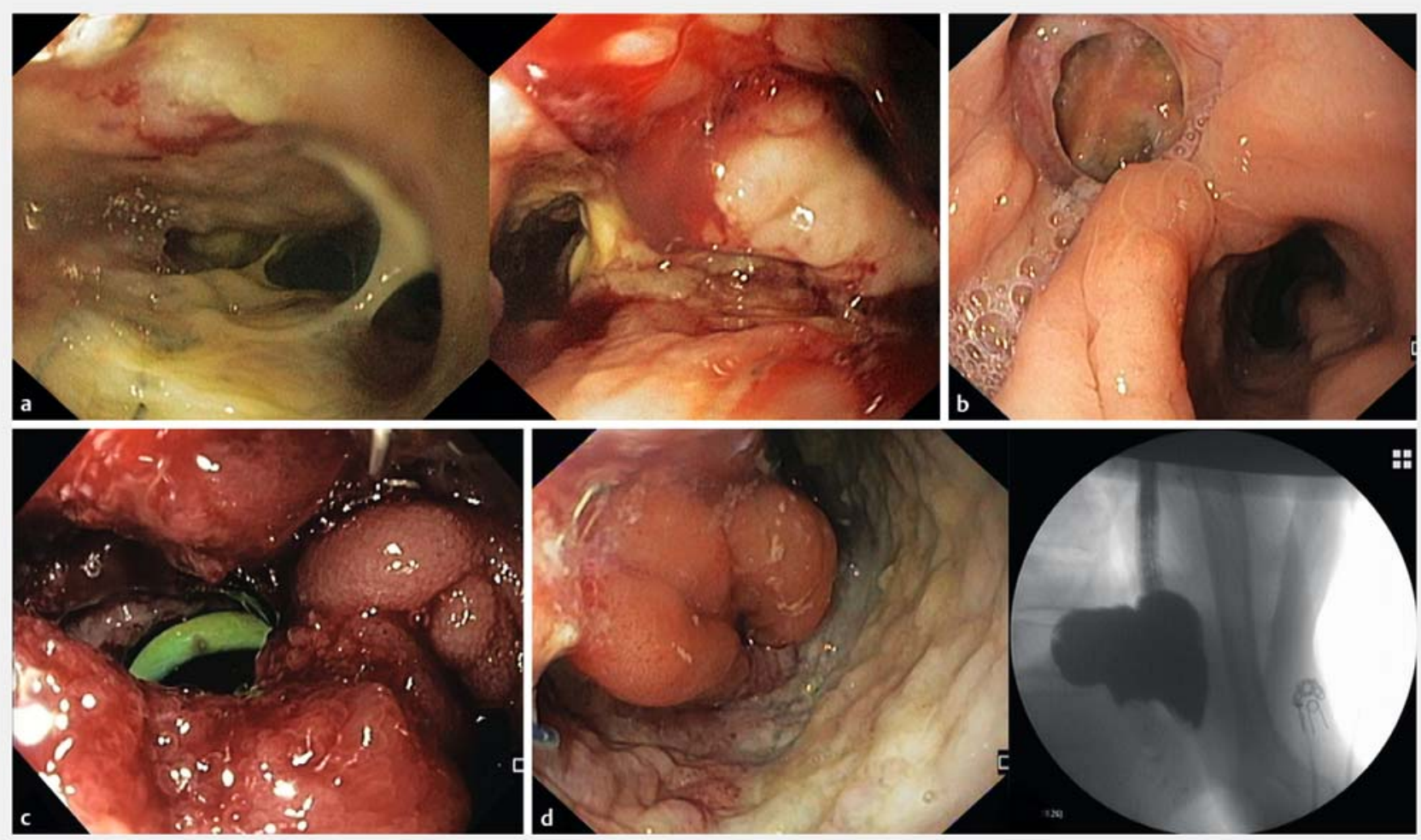

- Fig. 1 a Clinical case 1: 52-year-old man with subcutaneous emphysema and respiratory insufficiency after Ivor-Lewis esophagectomy due to esophagus squamous-cell carcinoma; chest CT with oral contrast revealed a $12-\mathrm{cm}$ intrathoracic collection with communication with the gastric tube; upper endoscopy revealed a severe anastomotic leakage $29 \mathrm{~cm}$ from the incisors. b Clinical case 2: 42-year-old woman, body mass index $38 \mathrm{~kg} / \mathrm{m}^{2}$, who underwent laparoscopic sleeve gastrectomy, without drain placement; 10 days later, she presented with a left pneumonia; chest CT with oral contrast revealed a 4-cm intrathoracic collection with communication with the gastric tube; upper endoscopy revealed a 20-mm anastomotic leakage $35 \mathrm{~cm}$ from the incisors; no stricture was present at the level of the incisura angularis. c Clinical case 3: 38-year-old man, with a body mass index of $40 \mathrm{~kg} / \mathrm{m}^{2}$, who underwent a Roux-en-Y gastric bypass, and presented 6 days later with fever and leukocytosis; CT with oral contrast revealed an $8 \mathrm{~cm}$ intraabdominal collection with communication with the gastric pouch; upper endoscopy revealed an anastomotic leakage $44 \mathrm{~cm}$ from the incisors; $\mathbf{d}$ Clinical case 4: 72-year-old man with recurrent leukocytosis and fever after total gastrectomy; CT with oral contrast revealed contrast extravasation between the gastrointestinal lumen and the intra-abdominal cavity; upper endoscopy revealed a severe anastomotic leakage $41 \mathrm{~cm}$ from the incisors. 
- Table 1 Techniques rating from the most frequently used to the less frequently used.

\begin{tabular}{|c|c|c|c|c|c|c|c|c|c|}
\hline & $\begin{array}{l}\text { First } \\
\text { most } \\
\text { used }\end{array}$ & $\begin{array}{l}\text { Second } \\
\text { most } \\
\text { used }\end{array}$ & $\begin{array}{l}\text { Third } \\
\text { most } \\
\text { used }\end{array}$ & $\begin{array}{l}\text { Fourth } \\
\text { most } \\
\text { used }\end{array}$ & $\begin{array}{l}\text { Fifth } \\
\text { most } \\
\text { used }\end{array}$ & $\begin{array}{l}\text { Sixth } \\
\text { most } \\
\text { used }\end{array}$ & $\begin{array}{l}\text { Seventh } \\
\text { most } \\
\text { used }\end{array}$ & Total & $\begin{array}{l}\text { Average } \\
\text { ranking }\end{array}$ \\
\hline Stent placement & $52.1 \%$ & $32.4 \%$ & $8.5 \%$ & $4.2 \%$ & - & $1.4 \%$ & - & 70 & 6.3 \\
\hline $\begin{array}{l}\text { Endoscopic vacuum } \\
\text { therapy }\end{array}$ & $15.5 \%$ & $14.1 \%$ & $7 \%$ & $7 \%$ & $8.5 \%$ & $15.5 \%$ & $11.3 \%$ & 56 & 4.1 \\
\hline Endoscopic suturing & $1.4 \%$ & $8.5 \%$ & $9.9 \%$ & $14.1 \%$ & $11.3 \%$ & $15.5 \%$ & $11.3 \%$ & 51 & 3.4 \\
\hline Tissue sealants & $7 \%$ & $5.6 \%$ & $11.3 \%$ & $14.1 \%$ & $15.5 \%$ & $21.1 \%$ & $12.7 \%$ & 62 & 3.4 \\
\hline Over-the-scope clips & $4.2 \%$ & $16.9 \%$ & $33.8 \%$ & $21.1 \%$ & $9.9 \%$ & $2.8 \%$ & $2.8 \%$ & 65 & 4.6 \\
\hline $\begin{array}{l}\text { Endoscopic septotomy } \\
\text { plus balloon dilation }\end{array}$ & $2.8 \%$ & $11.3 \%$ & $4.2 \%$ & $8.5 \%$ & $15.5 \%$ & $8.5 \%$ & $16.9 \%$ & 48 & 3.3 \\
\hline $\begin{array}{l}\text { Endoscopic internal } \\
\text { drainage }\end{array}$ & $16.9 \%$ & $9.9 \%$ & $21.1 \%$ & $16.9 \%$ & $14.1 \%$ & - & $2.8 \%$ & 60 & 4.7 \\
\hline Not applicable & - & $1.4 \%$ & $4.2 \%$ & $14.1 \%$ & $25.4 \%$ & $35.2 \%$ & $42.3 \%$ & & \\
\hline
\end{tabular}

consensus on the most appropriate therapeutic approach in management of UGI anastomotic leaks. The current study was designed to explore the current practices in the management of UGI anastomotic leaks of a panel comprising international expert therapeutic endoscopists with experience in leaks to help design and inform future prospective studies.

\section{Methods}

An online survey was developed to assess the opinion and practice of a panel of international expert therapeutic endoscopists regarding management of UGI anastomotic leaks. The participants were selected based on publications published on PubMed between January 2013 and April 2018 regarding endoscopic treatment of UGI anastomotic leaks. A total of 226 publications were found, corresponding to 182 different authors with available emails (first, last or corresponding authors). The survey was initially distributed, tested and optimized among 12 selected therapeutic endoscopists (ERP, AR, GD, GM, JD, JEvH, JMC, MGN, MS, PE, VK and MAK). Nineteen of the 182 obtained e-mail addresses were inactive. In August 2018, 163 participants were invited via an e-mail link to an online survey programme (http://www.surveymonkey.com), followed by a total of 3-weekly reminders.

The survey consisted of 35 opinion-probing questions (Appendix $\mathbf{1}$ ) and 4 short clinical cases ( $\bullet$ Fig. $\mathbf{1}$ ). With regard to the clinical vignettes, participants were asked to choose one option between the different endoscopic therapies available.

The final percentage in multiple-choice questions may exceed $100 \%$, as several respondents have chosen more than one answer. Average ranking (AR) in $>$ Table 1 and Supplementary Table 1 was calculated to determine which therapeutic choice was most preferred overall. It was calculated as follows, where " $w=$ weight of ranked position" and " $x=$ response count for answer choice"; weights are applied in reverse: $\frac{x_{1} w_{1}+x_{2} w_{2}+x_{3} w_{3} \ldots x_{n} w_{n}}{\text { Total }}$

Ideal patient characteristics for each endoscopic technique were based on the majority of respondents' answers ( $\bullet$ Table 2 ).

Data were collected non-anonymously and analyzed using the graphical and analytical features of www.surveymonkey. com and IBM SPSS Statistics, version 24.0 (IBM Corp., Armonk, New York, United States). Answers were described as counts and percentages for categorical variables. Continuous variables were summarized as medians and range. Regarding respondents who gave time ranges between treatment sessions, the median value of each range was used to determine the total median value.

This study was conducted according to the Declaration of Helsinki. All authors reviewed and approved the final manuscript.

\section{Results}

\section{Participants and endoscopic therapies characterization}

A total of 163 surveys were sent and 71 (44\%) were returned. Twenty-five countries on five different continents were represented. Sixty-nine percent of the respondents $(n=49)$ were gastroenterologists, with the remaining $31 \%(n=22)$ being surgeons. Eighty-two percent of respondents $(n=58)$ worked in academic hospitals and $18 \%(n=13)$ in non-academic teaching hospitals. The number of patients with anastomotic leaks treated within each therapeutic endoscopy unit in 1 year ranged from 1 to 4 at nine centers (13\%) to $>40$ at five hospitals $(7 \%)$ ( $\vee$ Fig. 2). Respondents had a median of 10 years $[1-36]$ of experience.

Placement of self-expandable metal stents (SEMS) was the technique most available in each department ( $97 \%$ ), followed by OTSC $(89 \%)$ and EID $(79 \%)$ ( $\bullet$ Fig. 3). Stent placement was 
- Table2 Ideal patient characteristics for each endoscopic technique.

\begin{tabular}{|c|c|c|c|c|c|c|}
\hline & \multicolumn{6}{|c|}{ Ideal patient characteristics ${ }^{1}$} \\
\hline & Stent & OTSC & EVT & Suture & Septotomy & EID \\
\hline \multicolumn{7}{|l|}{ Time of leak } \\
\hline - Acute & $93.8 \%$ & $96.8 \%$ & $48.7 \%$ & $89.5 \%$ & $3.2 \%$ & $54.3 \%$ \\
\hline - Chronic & $17.2 \%$ & $19 \%$ & $71.8 \%$ & $31.6 \%$ & $100 \%$ & $65.2 \%$ \\
\hline - $\mathrm{NO} / \mathrm{NI}$ & $n=7$ & $\mathrm{n}=8$ & $n=32$ & $n=33$ & $n=40$ & $n=25$ \\
\hline \multicolumn{7}{|l|}{ Leak size } \\
\hline . $0-1 \mathrm{~cm}$ & $54.1 \%$ & $77 \%$ & $25 \%$ & $64.7 \%$ & $51.9 \%$ & $63.6 \%$ \\
\hline . $1-2 \mathrm{~cm}$ & $63.9 \%$ & $47.5 \%$ & $40 \%$ & $50 \%$ & $63 \%$ & $65.9 \%$ \\
\hline . $2-3 \mathrm{~cm}$ & $55.7 \%$ & $9.8 \%$ & $67.5 \%$ & $47.1 \%$ & $51.9 \%$ & $45.5 \%$ \\
\hline - $>3 \mathrm{~cm}$ & $42.6 \%$ & - & $77.5 \%$ & $35.3 \%$ & $63 \%$ & $38.6 \%$ \\
\hline - $\mathrm{NO} / \mathrm{NI}$ & $n=10$ & $n=10$ & $n=31$ & $\mathrm{n}=37$ & $\mathrm{n}=44$ & $n=27$ \\
\hline \multicolumn{7}{|l|}{ Leak location } \\
\hline - Intrathoracic & $93.2 \%$ & $64 \%$ & $92.5 \%$ & $58.6 \%$ & $25 \%$ & $66 \%$ \\
\hline - Intraabdominal & $45.8 \%$ & $92 \%$ & $60 \%$ & $96.6 \%$ & $92.9 \%$ & $83 \%$ \\
\hline - $\mathrm{NO} / \mathrm{NI}$ & $n=12$ & $n=21$ & $n=31$ & $n=42$ & $n=43$ & $n=24$ \\
\hline \multicolumn{7}{|c|}{ Associated collection } \\
\hline - Yes & $11.3 \%$ & $7 \%$ & $95.2 \%$ & $11.1 \%$ & $90 \%$ & $97.9 \%$ \\
\hline - No & $88.7 \%$ & $93 \%$ & $4.8 \%$ & $88 n=35.9 \%$ & $10 \%$ & $2.1 \%$ \\
\hline - NO/NI & $n=9$ & $n=14$ & $n=29$ & & $n=41$ & $n=24$ \\
\hline \multicolumn{7}{|l|}{ Previous surgery } \\
\hline - Bariatric & $78.6 \%$ & $87.8 \%$ & $81.6 \%$ & $96.6 \%$ & $100 \%$ & $95.5 \%$ \\
\hline - Oncologic & $75 \%$ & $71.4 \%$ & $84.2 \%$ & $72.4 \%$ & $25.9 \%$ & $59.1 \%$ \\
\hline - $\mathrm{NO} / \mathrm{NI}$ & $n=15$ & $n=22$ & $n=33$ & $n=42$ & $n=44$ & $n=27$ \\
\hline
\end{tabular}

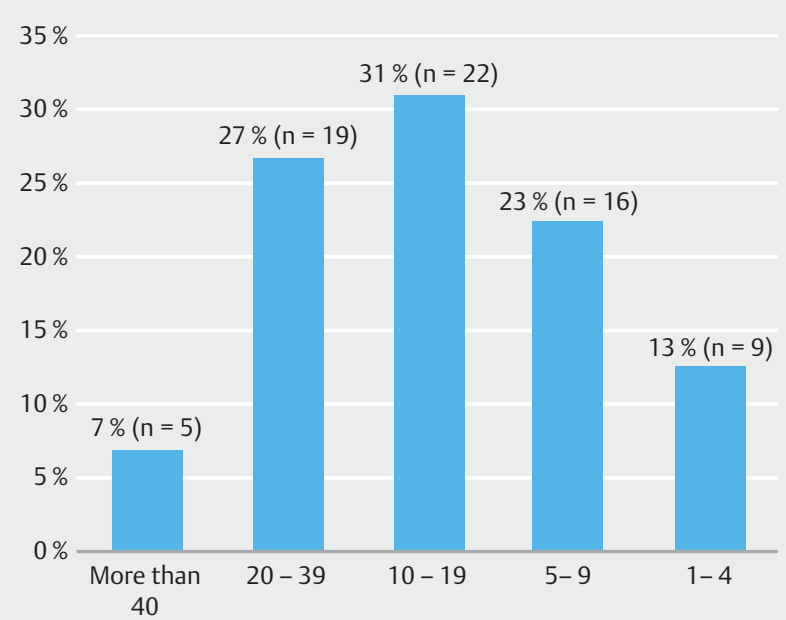

- Fig. 2 Respondents' answers to how many patients with anastomotic leaks does your therapeutic endoscopy unit usually treat in 1 year. the technique most frequently used (AR: 6.3), followed by EID (AR: 4.7), OTSC (AR: 4.6) and EVT (AR: 4.1) ( Table 1). AR for each continent, specialty, years of experience and patients treated per year are represented in Supplementary Table 1.

\section{Leaks characteristics}

Location (94\%), size (93\%), chronicity (91\%) and associated cavity $(90 \%)$ were the most relevant characteristics considered by respondents to impact choice of treatment. Sixty-five percent of the respondents treat bariatric leaks differently from oncologic surgery leaks. Collections not reachable by endoscopy $(69 \%)$ and insufficient internal drainage (66\%) were the most common indications for need for additional percutaneous/surgical drainage (Supplementary Table 2).

\section{Self-expandable stents}

Fifty-six percent of the 69 respondents with stent experience reported fully-covered SEMS (FC-SEMS) to be their usual first option, while $42 \%$ preferred partially-covered SEMS (PC- 


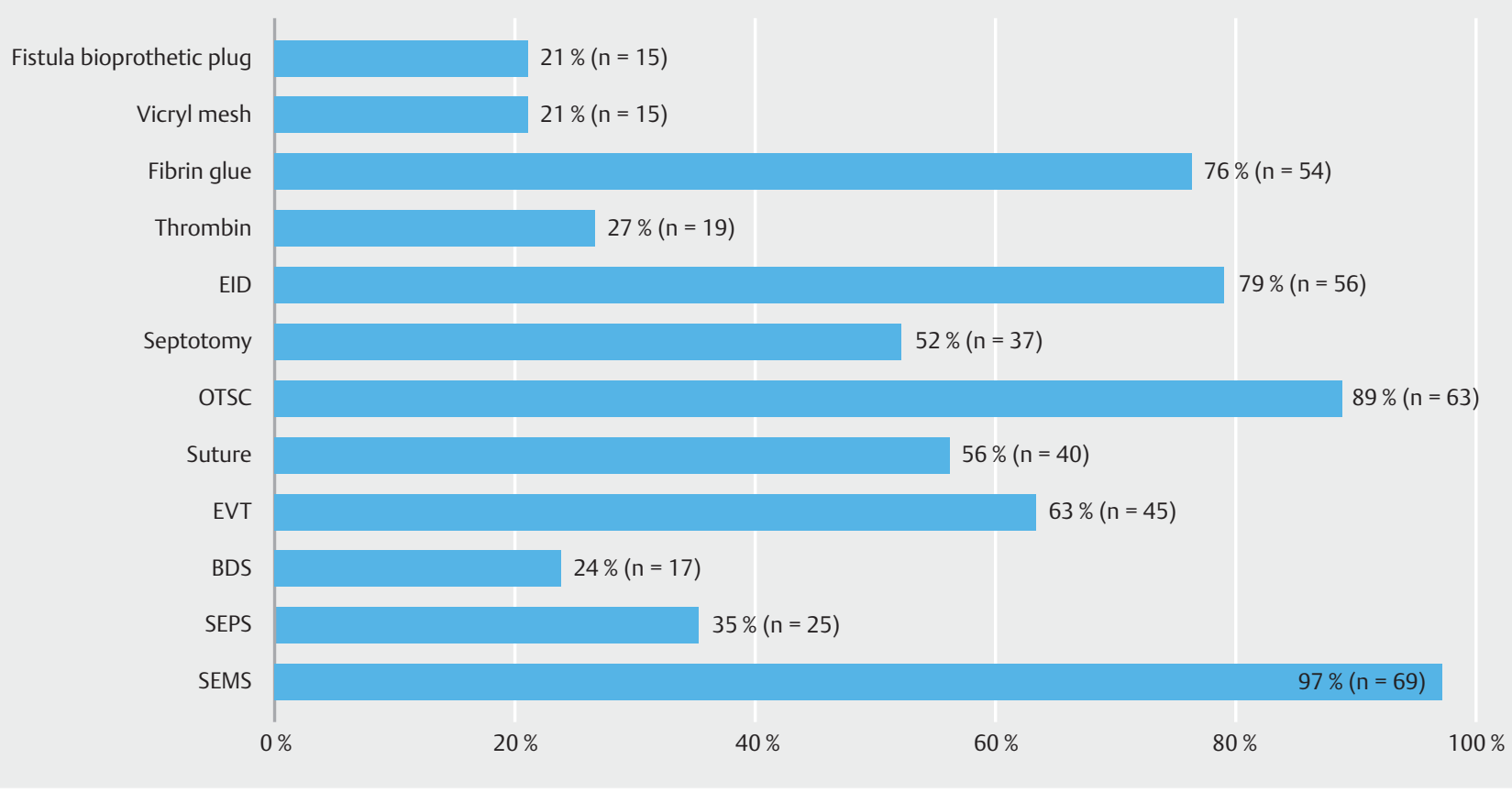

- Fig.3 Respondents' answers to techniques available in endoscopic departments.

SEMS). The majority ( $80 \%$ ) used techniques to minimize stent migration, with $38 \%(n=21)$ of them using combined therapies; placement of PC-SEMS is usually the preferred technique (45\%), followed by suture of the stent to the mucosa (33\%) and anchoring the stent with TTS clips (25\%) or OTSC (16\%).

Additional techniques to minimize stent migration are considered in patients with previous stent migration (52\%), if incomplete sealing between stent and esophageal wall (34\%) and when stents placed across jejunal anastomoses (19\%) ( Table 3).

The most common stent dwell time reported was 4 weeks (49\%) (range: 2 - 10 weeks) ( $\triangleright$ Table 3 ).

Patients with acute leaks (94\%), without associated collections (89\%), with intra-thoracic location (93\%) and less than $3 \mathrm{~cm}$ in diameter were considered ideal for stent placement; the majority of respondents considered both previous surgeries (bariatric or oncologic) suitable for stent placement ( $\triangleright$ Table 2).

\section{Over-the-scope clips}

Sixty-six percent and $37 \%$ of the 64 participants with OTSC experience reported placing them in acute and early leaks, respectively; $17 \%$ reported always performing epithelial ablation/damage prior to OTSC application, with $62 \%$ performing it at least in half of procedures ( $\downarrow$ Table 3 ).

Patients without associated collections (93\%), with intra-abdominal location (92\%), up to $1 \mathrm{~cm}$ in diameter (77\%) and resulting from previous bariatric surgery (88\%) were considered ideal patients for OTSC placement ( $\triangleright$ Table 2 ).

\section{Endoscopic vacuum therapy}

Seventy-five percent of the 40 respondents with EVT experience reported changing the polyurethane sponge every 3 to 5 days; $72 \%$ applied similar negative pressure for intra-thoracic and intra-abdominal leaks. Most commonly, negative pressures from 70 to $100 \mathrm{~mm} \mathrm{Hg}(41 \%)$ and 100 to $125 \mathrm{~mm} \mathrm{Hg}(\sim 35 \%)$ were used ( $\triangleright$ Table 4 ).

Regarding patients with large cavities but small leak defects, $56 \%$ performed balloon dilation and intracavitary EVT, while $28 \%$ placed the sponge intraluminally; $37 \%$ considered stent-over-sponge if difficulties in directing the vacuum force towards the leak, while $37 \%$ considered it to seal the sponge from the gastrointestinal lumen ( $\triangleright$ Table 4$)$.

Patients with chronic leaks (72\%), with associated collections (95\%), with intra-thoracic location (92\%) and with more than $2 \mathrm{~cm}$ in diameter were considered ideal for EVT therapy; the majority of respondents considered both previous surgeries suitable for EVT ( $\triangleright$ Table 2 ).

\section{Endoscopic suture}

Thirty-six percent of 36 respondents with suturing experience reported always performing epithelial ablation/damage prior to suturing, while $61 \%$ performed it in at least half of procedures ( $\triangleright$ Table 3 ).

Patients with acute leaks (89\%), without associated collections ( $89 \%$ ), up to $2 \mathrm{~cm}$ in diameter, with intra-abdominal location (97\%) and resulting from previous bariatric surgery (97\%) were considered ideal for endoscopic suturing ( $\triangleright$ Table 2 ). 
- Table 3 Respondents' answers to opinion probing questions regarding primary closure techniques.

\begin{tabular}{|c|c|c|c|}
\hline Technique & Question & Answer & $\%$ \\
\hline \multirow[t]{16}{*}{ Stents ${ }^{1}$} & \multirow[t]{3}{*}{ Self-expandable stent-first option } & Fully-covered & $56.5 \%$ \\
\hline & & Partially-covered & $42 \%$ \\
\hline & & Plastic & $1.4 \%$ \\
\hline & \multirow{4}{*}{$\begin{array}{l}\text { Techniques to minimize stent migration } \\
\text { (in patients without previous stent migration) }\end{array}$} & TTS clips/OTSC & $36.2 \%$ \\
\hline & & Suture & $33.3 \%$ \\
\hline & & PC-SEMS & $44.9 \%$ \\
\hline & & None & $20.3 \%$ \\
\hline & \multirow{5}{*}{$\begin{array}{l}\text { When to use additional techniques to minimize } \\
\text { stent migration? }\end{array}$} & Patients with previous stent migration & $52.2 \%$ \\
\hline & & Incomplete sealing between stent and esophageal wall & $34.3 \%$ \\
\hline & & Jejunal anastomoses & $19.4 \%$ \\
\hline & & Never & $11.9 \%$ \\
\hline & & Always & $25.4 \%$ \\
\hline & \multirow[t]{4}{*}{ Common stent dwell time } & 2 weeks & $6 \%$ \\
\hline & & 4 weeks & $49.3 \%$ \\
\hline & & 6 weeks & $28.4 \%$ \\
\hline & & $\geq 8$ weeks & $16.4 \%$ \\
\hline \multirow{11}{*}{$\begin{array}{l}\text { Over-the-scope } \\
\text { clips }^{2}\end{array}$} & \multirow[t]{5}{*}{ Time limit between leak and OTSC placement } & $<7$ days (acute leaks) & $65.6 \%$ \\
\hline & & 1 to 6 weeks (early leaks) & $37.5 \%$ \\
\hline & & 6 to 12 weeks (late leaks) & $6.3 \%$ \\
\hline & & >12 weeks (chronic leaks) & $9.4 \%$ \\
\hline & & Not relevant & $20.3 \%$ \\
\hline & \multirow[t]{6}{*}{ Epithelial ablation prior to OTSC placement } & Always & $17 \%$ \\
\hline & & $>90 \%$ of the cases & $20.8 \%$ \\
\hline & & $75 \%$ to $90 \%$ of the cases & $13.2 \%$ \\
\hline & & $50 \%$ to $75 \%$ of the cases & $11.3 \%$ \\
\hline & & $<50 \%$ of the cases & $20.8 \%$ \\
\hline & & Never & $17 \%$ \\
\hline \multirow{6}{*}{$\begin{array}{l}\text { Endoscopic } \\
\text { suture }^{3}\end{array}$} & \multirow[t]{6}{*}{ Epithelial ablation prior to suture } & Always & $36.1 \%$ \\
\hline & & $>90 \%$ of the cases & $16.7 \%$ \\
\hline & & $75 \%$ to $90 \%$ of the cases & $5.6 \%$ \\
\hline & & $50 \%$ to $75 \%$ of the cases & $2.8 \%$ \\
\hline & & $<50 \%$ of the cases & $25 \%$ \\
\hline & & Never & $13.9 \%$ \\
\hline \multicolumn{4}{|c|}{$\begin{array}{l}\text { OTSC, over-the-scope clip; PC-SEMS, partially covered self-expandable metal stent; TTS, through-the-scope } \\
{ }^{1} \text { Two to four endoscopists reported no experience with stents placement. } \\
{ }^{2} \text { Seven to } 18 \text { respondents reported no experience with OTSC placement. } \\
{ }^{3} \text { Thirty-five respondents reported no experience with endoscopic suture. }\end{array}$} \\
\hline
\end{tabular}




\section{Endoscopic septotomy}

Fifty-six percent of the 32 respondents with septotomy experience reported that the minimal time interval from surgery should be 4 weeks; $53 \%$ considered that limits of septotomy should be defined on a case-by-case basis. Regarding patients with previous sleeve gastrectomy, $13 \%$ always performed additional balloon dilation, while $81 \%$ only performed it if associated transgastric hyper-pressure. Ninety percent considered the need to perform further septotomy sessions, with presence of residual septum (50\%) and incomplete drainage (30\%) being the main indications; a median of 11 days $(6-35)$ between treatments was reported ( $>$ Table 4$)$.

Patients with chronic leaks (100\%), with associated collections (90\%), with intra-abdominal location (93\%) and resulting from previous bariatric surgery (100\%) were considered the ideal patients for endoscopic septotomy; all leak sizes were considered amenable to endoscopic septotomy ( $\triangleright$ Table 2 ).

\section{Endoscopic internal drainage}

The majority of respondents with EID experience reported preferring placement of two plastic stents ( $82 \%$ of 45 ) and shorter stents (62\% of 21 ) for drainage; $30 \%$ of 56 respondents referred to never performing necrosectomy. A median of 14 days (1$90)$ between stents exchange was reported. A median of 4.5 days $(0-42)$ until oral diet resumption was reported, with $21 \%$ of respondents $(n=9)$ starting the day of procedure or day after ( $\triangleright$ Table 4).

Patients with chronic leaks (65\%), with associated collections (97\%), with intra-abdominal location ( $83 \%$ ), up to $2 \mathrm{~cm}$ in diameter and resulting from previous bariatric surgery (95\%) were considered ideal for endoscopic internal drainage ( Table 2).

\section{Endoscopic failure}

Persistent inflammation with clinical sepsis (55\%) was the definition most commonly reported for endoscopic failure, followed by inability to resume oral feeding (42\%), duration of treatment (39\%), chronic reepithelized fistula (37\%), number of endoscopic sessions (30\%) and closure not achieved after 1 month of treatment with one single technique (28\%).

\section{Clinical cases}

EVT was the therapeutic option most often chosen (27\%) in post Ivor-Lewis esophagectomy with an intra-thoracic associated collection (clinical case 1), followed by stent placement plus drainage (23\%) and stent placement (14\%) ( $>$ Fig. 4a); EVT and EID were the therapeutic options most often chosen in postsleeve gastrectomy $2 \mathrm{~cm}$ in diameter (clinical case 2) and post RYGB with an intraabdominal associated collection (clinical case 3 ) (21\% and $20 \%$, respectively), followed by stent placement plus drainage ( $17 \%$ and $14 \%$ ) ( $\triangleright$ Fig. 4b, $>$ Fig. 4c); surgery was the therapeutic option most often chosen in post total gastrectomy (clinical case 4 ) (24\%), followed by stent placement with or without drainage (19\%) ( $\triangleright$ Fig.4d).

\section{Discussion}

Therapeutic endoscopy plays a major role in management of UGI anastomotic leaks, offering an effective treatment alternative to repeat surgery [10]. The available endoscopic approaches range from primary to secondary closure techniques, with varying degrees of technical and clinical success and adverse events, generating a lack of consensus regarding the most appropriate endoscopic management [11].

This survey shows that placement of stents, specifically SEMS, is the technique most available and most frequently used in almost every department. Even though OTSC are also well-represented, they are not a common first option, as represented in the clinical cases section. This is probably related to the need for pliable tissue for successful placement, as well as risk of leak recurrence due to OTSC displacement $[8,12]$. Other reasons for failed closure may be related to poor integrity of the tissue surrounding the leak as a result of ischemia and inflammation as well as poor/partial placement over often large defects. On the other hand, EID and EVT seem to be increasingly used techniques; this is probably related to the fact that closing leaks with tissue apposition techniques or diversion therapy does not seem to be the ideal treatment strategy in some cases, especially in late or chronic leaks. EVT and EID allow optimal drainage of the cavity, ensuring granulation, utilizing the concept of keeping the leak open [12].

The majority of participants considered that bariatric leaks should be treated differently from oncologic leaks, with location, size, chronicity, and associated cavity being the most relevant leak characteristics. Need for additional percutaneous/surgical drainage is almost always considered when closure techniques (tissue apposition or diversion techniques) are chosen, as internal drainage is not achieved, either by the collection not being reachable $(69 \%)$ or sealed $(66 \%)$. All of these is reflected in clinical cases choices. EVT and stent placement, with or without percutaneous/surgical drainage, were the therapeutic options most often chosen in patients with previous oncologic surgery, while EVT and EID were the therapeutic options most often chosen in patients with previous bariatric surgery. Interestingly, surgery was the first option in post-total gastrectomy case ( $24 \%$ of respondents), despite no previous endoscopic treatment failure nor presence of uncontained leak. This might be explained by the almost complete leak of the anastomosis.

Regarding self-expandable stents, both FC (56\%) and PCSEMS (42\%) were similarly selected as first options, even in patients without previous stent migration; besides PC-SEMS, 35\% of respondents used other additional techniques to minimize stent migration (in patients without previous stent migration), with endoscopic suturing of FC-SEMS being the preferred technique, as it seems to lower rates of stent migration [13]. Optimal duration of stent dwell is unknown and is likely related to leak classification and size as well as patient-related factors [14]. Stent dwell time ranged from 4 to 6 weeks in $77 \%$ of respondents.

Regarding endoscopic suturing, respondents believed it provides the ability to close larger defects than OSTC $(2 \mathrm{~cm}$ versus 
- Table4 Respondents' answers to opinion probing questions regarding secondary closure techniques.

\begin{tabular}{|c|c|c|c|c|}
\hline Technique & Question & \multicolumn{2}{|l|}{ Answer } & $\%$ \\
\hline \multirow{14}{*}{$\begin{array}{l}\text { Endoscopic } \\
\text { vacuum therapy }{ }^{1}\end{array}$} & \multirow{3}{*}{$\begin{array}{l}\text { Approach in patients with large } \\
\text { cavities but small leak defects }\end{array}$} & \multicolumn{2}{|c|}{ Intraluminal EVT } & $28.2 \%$ \\
\hline & & \multicolumn{2}{|c|}{ Balloon dilation and intracavitary EVT } & $56.4 \%$ \\
\hline & & \multicolumn{2}{|c|}{ EVT plus stent } & $15.4 \%$ \\
\hline & \multirow[t]{4}{*}{ How often change sponge in EVT } & \multicolumn{2}{|l|}{$<3$ days } & $5 \%$ \\
\hline & & \multicolumn{2}{|c|}{ Every 3 to 5 days } & $75 \%$ \\
\hline & & \multicolumn{2}{|c|}{ Every 5 to 7 days } & $15 \%$ \\
\hline & & \multicolumn{2}{|c|}{ Case by case } & $5 \%$ \\
\hline & \multirow{4}{*}{$\begin{array}{l}\text { Negative pressure for intra- } \\
\text { thoracic/intra-abdominal leaks }\end{array}$} & \multicolumn{2}{|c|}{$<70 \mathrm{~mm} \mathrm{Hg}$} & $16.2 \%$ \\
\hline & & \multicolumn{2}{|c|}{$70 \mathrm{~mm}$ to $100 \mathrm{~mm} \mathrm{Hg}$} & $40.5 \%$ \\
\hline & & \multicolumn{2}{|c|}{$100 \mathrm{~mm}$ to $125 \mathrm{~mm} \mathrm{Hg}$} & $35.9 \% / 35.1 \%$ \\
\hline & & \multicolumn{2}{|c|}{$>125 \mathrm{~mm} \mathrm{Hg}$} & $7.7 \% / 8.1 \%$ \\
\hline & \multirow[t]{3}{*}{ When stent-over-sponge } & \multicolumn{2}{|c|}{ If difficulties in directing vacuum force towards the leak } & $36.7 \%$ \\
\hline & & \multicolumn{2}{|c|}{ To seal the sponge from the gastrointestinal lumen } & $36.7 \%$ \\
\hline & & \multicolumn{2}{|l|}{ Never } & $43.3 \%$ \\
\hline \multirow{15}{*}{$\begin{array}{l}\text { Endoscopic } \\
\text { septotomy }\end{array}$} & \multirow{3}{*}{$\begin{array}{l}\text { When perform additional balloon } \\
\text { dilation }\end{array}$} & \multicolumn{2}{|c|}{ If associated transgastric hyper-pressure (stricture/twist) } & $80.6 \%$ \\
\hline & & \multicolumn{2}{|l|}{ Always } & $12.9 \%$ \\
\hline & & \multicolumn{2}{|l|}{ Never } & $6.5 \%$ \\
\hline & Minimal time interval since & \multicolumn{2}{|l|}{2 weeks } & $15.6 \%$ \\
\hline & & 4 weeks & & $56.3 \%$ \\
\hline & & $>6$ week & & $28.1 \%$ \\
\hline & Limits of septotomy & Cavity le & & $47.1 \%$ \\
\hline & & Case-by- & & $52.9 \%$ \\
\hline & Need for further sessions & Yes & & $90 \%$ \\
\hline & & No & & $10 \%$ \\
\hline & When further situations & Leak clea & & $30 \%$ \\
\hline & & Residual & & $50 \%$ \\
\hline & & If cavity $i$ & & $5 \%$ \\
\hline & & Larger co & & $15 \%$ \\
\hline & Time between sessions & Median, & & 11 days $(6-35)$ \\
\hline Endoscopic & When to perform necrosectomy & Always & & $5.4 \%$ \\
\hline 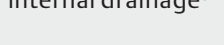 & & If presen & & $64.3 \%$ \\
\hline & & Never & & $30.4 \%$ \\
\hline & Which stents & Number & Single & $11.1 \%$ \\
\hline & & & Double & $82.2 \%$ \\
\hline & & & One or the other & $6.7 \%$ \\
\hline & & Length & Shorter & $61.9 \%$ \\
\hline & & & Longer & $33.3 \%$ \\
\hline & & & One or the other & $4.8 \%$ \\
\hline & Time between sessions & Median, & & 14 days $(1-90)$ \\
\hline & Time until oral diet resumption & Median, & & 4.5 days $(0-42)$ \\
\hline & & End of $\operatorname{tr}$ & & $12.5 \%$ \\
\hline
\end{tabular}

EVT, endoscopic vacuum therapy

${ }^{1}$ Thirty-one to 41 endoscopists reported no experience with EVT.

2 Seven to 41 endoscopists reported no experience with endoscopic septotomy.

${ }^{3}$ Five to 29 endoscopists reported no experience with endoscopic internal drainage. 


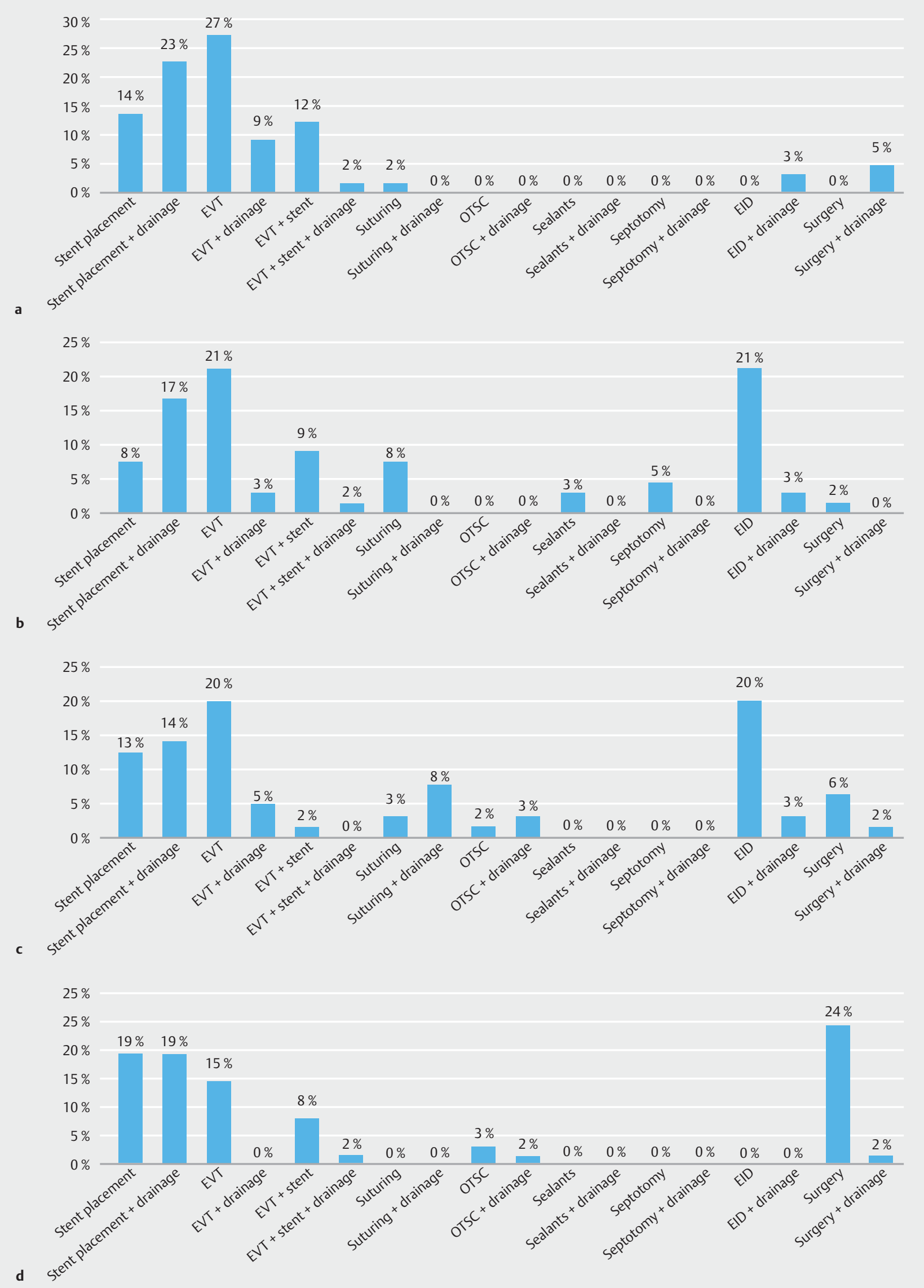

- Fig.4 Respondents' answers to clinical cases section. 
$1 \mathrm{~cm})$. Both require robust mucosa to hold the sutures when tissue is pulled in apposition [8]; epithelial ablation/damage to the defect edges before OTSC or suturing may increase procedure success and result in a more durable seal, with $\sim 60 \%$ of respondents performing it in more than half of procedures.

Regarding EVT, sponges can be placed intracavitary and/or intraluminal, depending on the defect size and presence of an extraluminal cavity [1]. Even though intraluminal EVT might be easier and safer than intracavitary EVT [12], the majority (56\%) of respondents preferred to perform balloon dilation and intracavitary EVT, as leak closure might be better [12]. One of the great disadvantages of EVT is the need for repeat endoscopic procedures, as the majority of respondents (90\%) changed the polyurethane sponge every 3 to 5 days or 5 to 7 days.

Similar to peripancreatic collections drainage, an organized walled-off collection must be established for endoscopic septotomy to be safe and effective. This was reflected by the majority of respondents (56\%), who only consider it at least 4 weeks after surgery. Management of the downstream stenosis within the sleeve that creates an unfavorable pressure gradient was also considered critical to enhance drainage and correction of one of the underlying physiologic defects that predisposed and perpetuated the leak. Need for repeated septotomy (90\%) was mostly based on presence of residual septum (50\%) or incomplete clearance of the cavity (30\%).

Although EID with transgastric stents appears to be effective, controversies exist regarding optimal technique [15]. Even though necrosectomy may expedite clinical improvement, $30 \%$ of respondents reported never doing it. A median of 4.5 days until oral diet resumption was reported, with $21 \%$ starting it the day of or after procedure, as it is believed oral contents do not enter the perigastric cavity. Regarding stent exchange, while some saw no value in routine stent exchange unless necrosectomy was also performed [15], performance of multiple procedures may allow to evaluate treatment progression to adapt internal drainage, as well as promote healing by inducing trauma in the pseudocavity with exchange of the pigtail stents [16].

As there are no comparative studies between the different endoscopic techniques, it is difficult to establish a therapeutic algorithm in these patients. Determining optimal therapy for such patients requires careful examination of patient clinical status, anastomotic defect, and a review of all available options, local expertise, and previous experience. The approach to UGI anastomotic leaks should always be individualized and multidisciplinary. Considering the majority of respondents' answers, acute and small leaks without associated collections may be considered for stent placement (up to $3 \mathrm{~cm}$ ), OSTC placement (up to $1 \mathrm{~cm}$ ) or endoscopic suture (up to $2 \mathrm{~cm}$ ). In the setting of associated collection, these techniques can still be considered if external drainage is also performed; if not, EVT, EID and endoscopic septotomy should be considered, with EVT and EID being an option in acute and chronic leaks, while endoscopic septotomy should only be performed in leaks with more than 4 weeks' duration. While endoscopic septotomy can be considered for all leak sizes, EID is ideal for leaks up to $2 \mathrm{~cm}$ and EVT for leaks larger than $2 \mathrm{~cm}$. Intrathoracic leaks may be better served with stents or EVT, and intraabdominal leaks with OTSC, suturing, septotomy or EID. Leaks resulting from previous bariatric surgery should ideally be treated with OTSC, suture, septotomy or EID, while stents and EVT can be considered for leaks related to bariatric and oncologic surgeries.

\section{Conclusion}

In conclusion, this study provides an overview of the techniques used for endoscopic management of UGI leaks and shows that there is wide variation in management of patients with UGI anastomotic leaks, even among the most expert in the field, particularly concerning difficult-to-treat patients, possibly reflecting the poor quality of evidence available at the moment. Limitations of our study include a survey response rate of only $44 \%$ which may subject the study to bias, making interpretation of results more challenging. However, this study presents information which to date has not been available, with inclusion of experts from various countries, different opinion questions regarding each technique, and different clinical scenarios. Even though there is no consensus on the definition of endoscopic failure, persistent inflammation with clinical sepsis and impossibility to resume oral feeding should be strongly considered. Future prospective studies should address these issues, and for which transnational collaborations are urgently needed, so that we move from an expert- to an evidence- and personalizationbased care in endoscopic treatment of upper anastomotic leaks.

\section{Full list of collaborators ordered alphabetically}

Abraham Mathew ${ }^{1}$, Alberto Fernández Villaverde², Alfredo Daniel Guerron ${ }^{3}$, Andrea Tringali ${ }^{4}$, Bram Vermeulen ${ }^{5}$, Brian Louie $^{6}$, Bruno Meduri ${ }^{7}$, Carmelo Luigiano ${ }^{8}$, Christoph Gubler ${ }^{9}$, Diane Lorenzo $^{10}$, Eric M Pauli ${ }^{1}$, Giorgio Baretta ${ }^{11}$, Gottumukkala S Raju ${ }^{12}$, Gunnar Loske ${ }^{13}$, Guy Pines ${ }^{14}$, Haicam El Mourad ${ }^{15}$, Hany Shehab $^{16}$, Hideki Kobara ${ }^{17}$, Hyuk Lee ${ }^{18}$, Hyunsoo Chung ${ }^{19}$ I-Rue Lai $^{20}$, Ioannis Rouvelas ${ }^{21}$, Jean-Michel Gonzalez ${ }^{10}$, Joan B Gornals $^{22}$, Jochen Wedemeyer ${ }^{23}$, John M DeWitt ${ }^{24}$, Joo Young $\mathrm{Cho}^{25}$, Katerina Kotzampassi26, Ke-Neng Chen ${ }^{27}$, Lee L Swanström ${ }^{28}$, Majid A Almadi ${ }^{29}$, Marc Barthet ${ }^{10}$, María-Victoria Alvarez-Sánchez ${ }^{30}$, Mati Shnell ${ }^{31}$, Michael A Schweitzer ${ }^{32}$, Michael Christian Sulz ${ }^{33}$, Michael G Sarr ${ }^{34}$, Michael Talbot ${ }^{35}$, Milena Di Leo $^{36}$, Mukesh Nasa ${ }^{37}$, Nick J Newton ${ }^{38}$, Nils Heits ${ }^{39}$, Paolo Giorgio Arcidiacono ${ }^{40}$, Pawel Rogalski ${ }^{41}$, Pedro Pereira ${ }^{42}$, Peter Bauerfeind $^{9}$, Peter Siersema ${ }^{5}$, Piyush Ranjan ${ }^{43}$, Radu Pescarus ${ }^{44}$, Rahul Pannala ${ }^{45}$, Ravinder Ogra ${ }^{46}$, Richard Kozarek ${ }^{47}$, Ryan Law $^{48}$, Shanda H Blackmon ${ }^{49}$, Shayan Irani ${ }^{47}$, Sigal Fishman ${ }^{31}$, Steven G Leeds ${ }^{50}$, Tolga Erim ${ }^{51}$, Victoria Gómez ${ }^{52}$

1 Section of Minimally Invasive and Bariatric Surgery, Department of Surgery, Penn State Milton S. Hershey Medical Center, Hershey, Pennsylvania, United States

2 Gastroenterology Department, POVISA Hospital, Vigo, Pontevedra, Spain

${ }^{3}$ Division of Metabolic and Weight Loss Surgery, Department of 
Surgery, Duke University, Durham, North Carolina, United States

${ }^{4}$ Digestive Endoscopy Unit. IRCCS, Fondazione Policlinico Universitario A. Gemelli, Catholic University, Rome, Italy

${ }^{5}$ Department of Gastroenterology and Hepatology, Radboud University Medical Center, Nijmegen, The Netherlands

${ }^{6}$ Division of Thoracic Surgery, Swedish Cancer Institute and Medical Center, Seattle, Washington, United States

7 Department of Gastroenterology and Hepatology, Hôpital Privé des Peupliers, Paris, France

${ }^{8}$ Digestive Endoscopy Unit, San Paolo Hospital, Milan, Italy9 Division of Gastroenterology and Hepatology, University Hospital Zürich, Zürich, Switzerland

10 Department of Gastroenterology, Aix Marseille Univ-APHMHôpital Nord, Marseille, France

${ }^{11}$ Federal University of Parana, Curitiba, Parana, Brazil

12 The University of Texas Graduate School of Biomedical Sciences at Houston and MD Anderson Cancer Center, Houston, Texas, United States

13 Department for General, Abdominal, Thoracic and Vascular Surgery, Katholisches Marienkrankenhaus Hamburg gGmbH, Hamburg, Germany

14 Department of Surgery, Kaplan Medical Center, Rehovot and The Hebrew University School of Medicine, JerUnited Stateslem, Israel

${ }^{15}$ Department of Bariatric Surgery, AZ Sint Blasius Medical Center, Dendermonde, Belgium

${ }^{16}$ Gastrointestinal Endoscopy Unit, Department of Gastroenterology, Cairo University, Cairo, Egypt

17 Department of Gastroenterology and Neurology, Faculty of Medicine, Kagawa University, Takamatsu, Japan

18 Department of Medicine, Samsung Medical Center, Sungkyunkwan University School of Medicine, Seoul, Republic of Korea

${ }^{19}$ Division of Gastroenterology, Department of Internal Medicine and Liver Research Institute, Seoul National University College of Medicine, Seoul, Republic of Korea

${ }^{20}$ Department of Surgery, National Taiwan University Hospital, Taipei, Taiwan

${ }^{21}$ Centre for Digestive Diseases, Karolinska University Hospital and Division of Surgery, Department of Clinical Intervention and Technology (CLINTEC), Karolinska Institutet, Stockhom, Sweden

22 Endoscopy Unit, Department of Digestive Diseases, Hospital Universitari de Bellvitge, Bellvitge Biomedical Research Institute (IDIBELL), University of Barcelona, Barcelona, Spain

23 Department of Gastroenterology, Hepatology and Endocrinology, Medical School Hannover, Hannover, Germany

24 Division of Gastroenterology \& Hepatology, Indiana University, Indianapolis, Indiana, United States

${ }^{25}$ Department of Gastroenterology, Cha Bundang Medical Center, Cha University College of Medicine, Seongnam-si, South Korea

26 Department of Surgery, Aristotle University of Thessaloniki, AHEPA Hospital, Thessaloniki, Greece

27 Department of Thoracic Surgery I, Beijing University Cancer Hospital, Beijing, China
${ }^{28}$ Gastrointestinal and Minimally Invasive Surgery, The Oregon Clinic, Portland, Oregon, United States

${ }^{29}$ Division of Gastroenterology, King Khalid University Hospital, King Saud University, Riyadh, Saudi Arabia

30 Department of Gastroenterology, Complejo Hospitalario de Pontevedra, Pontevedra, Spain

31 Obesity Service, Department of Gastroenterology and Liver Diseases, Tel Aviv Medical Center, affiliated with Sackler School of Medicine, Tel Aviv university, Tel Aviv, Israel

32 Department of Surgery, Johns Hopkins Medical Institutions, Baltimore, MD, United States

33 Division of Gastroenterology, Kantonsspital St. Gallen, St. Gallen, Switzerland

${ }^{34}$ Department of Gastroenterologic and General Surgery, Mayo Clinic, Rochester, Minnesota, United States

35 Department of Surgery, University of New South Wales, St George Hospital, Kogarah, New South Wales, Australia

${ }^{36}$ Digestive Endoscopy Unit, Humanitas Research Hospital, Rozzano, Milan;Humanitas University, Rozzano, Milan, Italy

37 Institute of Digestive and Hepatobiliary Sciences, Medanta The Medicity, Gurgaon, Delhi Near Rajiv Chowk, India

38 Gastrointestinal Services, University College Hospital, London

39 Department of General, Visceral, Thoracic, Transplantation and Pediatric Surgery, University Hospital Schleswig-Holstein, Kiel, Germany

40 Pancreato-Biliary Endoscopy and Endosonography Division, Pancreas Translational and Clinical Research Center, IRCCS San Raffaele Scientific Institute, Vita-Salute San Raffaele University, Milan, Italy

41 Department of Gastroenterology and Internal Medicine, Medical University of Bialystok, Bialystok, Poland

42 Gastroenterology Department, Centro Hospitalar São João, Porto, Portu

43 Institute of Liver, Gastroenterology, and Pancreaticobiliary Sciences, Sir Ganga Ram Hospital, Rajinder Nagar, New Delhi, India

${ }^{44}$ Département de Chirurgie, Division de Chirurgie Bariatrique, Hôpital du Sacré-Coeur de Montréal, Université de Montréal, Montréal, Canada

45 Division of Gastroenterology and Hepatology, Departments of Medicine, Mayo Clinic, Scottsdale, Arizona, United States

46 Department of Gastroenterology, Middlemore Hospital, Auckland, New Zealand

47 Digestive Disease Institute, Virginia Mason Medical Center, and University of Washington, Seattle, Washington, United States

48 Division of Gastroenterology, University of Michigan, Ann Arbor, Michigan, United States

${ }^{49}$ Division of General Thoracic Surgery, Mayo Clinic, Rochester, Minnesota, United States

${ }^{50}$ Division of Minimally Invasive Surgery, Baylor University Medical Center at Dallas, Texas, United States

51 Department of Gastroenterology and Hepatology, Cleveland Clinic Florida, Weston, Florida

52 Division of Gastroenterology and Hepatology, Mayo Clinic, Jacksonville, Florida, United States 


\section{Competing interests}

Alessandro Repici is a consultant for Boston Scientific. Jacques Devière is a consultant for Boston Scientific and Olympus. Jeanin E. van Hooft is a consultant for Medtronic and Boston Scientific and has a research grant from Cook Medical and Abbott. Manoel Galvao Neto is a consultant for Olympus, Ethicon Endosurgery, Apollo Endosurgery, Meditronics, Fractyl Laboratories, GI Dynamics, GI Windows, Alacer Biomedica, CMS/Sci-Tech, M.I.Tech and NitiNotes. Pierre Eisendrath received a research grant from Endo Tools Therapeutics. Vivek Kumbhari is a consultant for Apollo Endosurgery, Boston Scientific, Medtronic, Pentax Medial, ReShape Lifesciences and receives research support from ERBE and Apollo Endosurgery. Mouen A. Khashab is a consultant for Boston Scientific, Olympus and Medtronic. None of the authors disclosed personal conflicts of interest or financial relationships relevant to this publication.

\section{References}

[1] Bemelman WA, Baron TH. Endoscopic management of transmural defects (leaks/perforations/fistulae). Gastroenterology 2018; 154: 1938 - 1946.e1

[2] Rutegård M, Lagergren P, Rouvelas I et al. Intrathoracic anastomotic leakage and mortality after esophageal cancer resection: a population-based study. Ann Surg Oncol 2012; 19: 99-103

[3] Lang H, Piso P, Stukenborg C et al. Management and results of proximal anastomotic leak in a series of 1114 total gastrectomies for gastric carcinoma. Eur J Surg Oncol 2000; 26: 168-71

[4] Messager M, Warlaumont M, Renaud F et al. Recent improvements in the management of esophageal anastomotic leak after surgery for cancer. Eur J Surg Oncol 2017; 43: 258-269
[5] Blencowe NS, Strong S, McNair AG et al. Reporting of short-term clinical outcomes after esophagectomy: a systematic review. Ann Surg 2012; 255: 658-66

[6] Morales MP, Miedema BW, Scott JS et al. Management of postsurgical leaks in the bariatric patient. Gastrointest Endosc Clin N Am 2011; 21: $295-304$

[7] Gonzalez R, Sarr MG, Smith CD et al. Diagnosis and contemporary management of anastomotic leaks after gastric bypass for obesity. J Am Coll Surg 2007; 204: 47-55

[8] Cho J, Sahakian AB. Endoscopic Closure of Gastrointestinal Fistulae and Leaks. Gastrointest Endosc Clin N Am 2018; 28: 233 - 249

[9] Di Leo M, Maselli R, Ferrara EC et al. Endoscopic management of benign esophageal ruptures and leaks. Curr Treat Options Gastroenterol 2017; 15: $268-284$

[10] Eisendrath P, Devière J. Digestive leaks: An approach tailored to both indication and anatomy. Endosc Int Open 2016; 4: E652 - E653

[11] Vargas E], Abu Dayyeh BK. Keep calm under pressure: a paradigm shift in managing postsurgical leaks. Gastrointest Endosc 2018; 87: 438 441

[12] Rodrigues-Pinto E, Morais R, Macedo G et al. Choosing the appropriate endoscopic armamentarium for treatment of anastomotic leaks. Am J Gastroenterol 2019; 114: 367-371

[13] Bick BL, Imperiale TF, Johnson CS et al. Endoscopic suturing of esophageal fully covered self-expanding metal stents reduces rates of stent migration. Gastrointest Endosc 2017; 86: 1015- 1021

[14] Kumbhari V, Cai JX, Schweitzer MA. Endoscopic management of bariatric surgical complications. Curr Opin Gastroenterol 2015; 31: 359 367

[15] Kumbhari V, Abu Dayyeh BK. Keeping the fistula open: paradigm shift in the management of leaks after bariatric surgery? Endoscopy 2016; 48: $789-791$

[16] Donatelli G, Dumont JL, Cereatti F et al. Endoscopic internal drainage as first-line treatment for fistula following gastrointestinal surgery: a case series. Endosc Int Open 2016; 4: E647-E651 